Journal of Engineering and Applied Sciences 15 (6): 1515-1521, 2020

ISSN: 1816-949X

(C) Medwell Journals, 2020

\title{
Influence of Hammer Properties on Upper-Extremity Movement Variance during Hammering
}

\author{
${ }^{1}$ Langenderfer Joseph E., ${ }^{1}$ Ferdous Sumaya and ${ }^{2}$ Balendra Nilanthy \\ ${ }^{1}$ School of Engineering and Technology, \\ ${ }^{2}$ Department of Physical Therapy, Central Michigan University, \\ Mount Pleasant, Michigan 48859, USA
}

\begin{abstract}
The objective of this study was to characterize the variance of hammering movement and to determine movement dimensionality as related to hammer properties. Positions of markers attached to upper-extremity and hammers were recorded while 19 subjects hammered nails with four different hammers. Principal movements were calculated with principal components analysis. The principal movements differed between hammers and subjects used different principal movements indicating different movement strategies. Contributions of principal movements to variance and differences in movement dimensionality were determined between hammers and across subjects. Long handle hammers resulted in lower movement dimension as four principal movements were required to explain $95 \%$ of variance. For short handle hammers five principal movements were required to explain the same variance. Differences in explained variance and dimensionality provide insight into effects of hammers with different properties on strategies for movement and demands on motor control and musculoskeletal systems.
\end{abstract}

Key words: Kinematics, motion, strategy, PCA, variance

\section{INTRODUCTION}

Swinging a hammer is an important task for performing work and results in a repetitive, highly variable, multi-joint movement with high potential for fatigue and overuse injuries for the upper-extremity. Hammering to fatigue has been shown to effect motion of the elbow and trunk but not the wrist or shoulder (Cote et al., 2005) with decreases in elbow motion found to be associated with increases in trunk motion. In the same study, shoulder injured subjects for whom the hammering task was likely more challenging were found to hammer with a less variable inter-joint coordination with decreased variability in time delays from time of hammer hit to maximum joint angular velocities. As different types of hammers exist and the human must interact with and control the hammer, hammer properties may also influence organization and variability of the movement (Carello and Wagman, 2009).

The role of hammer properties in influencing hammer effectiveness has been investigated. When subjects used toy hammers with greater inertia relative to the balance (or symmetry) of inertia, they were able to more effectively pound pegs as measured by the number of pegs driven. Additionally, when inertia symmetry was increased relative to overall inertia, subjects compensated by increasing the amplitude of vertical hammer translation. A generalization has been developed that objects with larger inertia require greater torque and muscle forces while the symmetry of the inertia affects the diversity and patterning of the torque and forces (Carello and Wagman, 2009; Fitzpatrick et al., 2015). Hammer properties may also influence the variance of upper-extremity movement. However, these prior studies only measured hammer movements, so, little is known on how upper-extremity movement may be affected when using different hammers. More recently, few differences in hammer motion amplitude, timing and hammer force exerted on ground were found when swinging hammers of different mass and face size (Petric et al., 2017), leading to the conclusion of motor control robustness to minor changes in dynamics from different hammers resulting in similar swinging. While different hammers may be swung in a manner that from the external appears to be conserved, there may be subtle differences in how the movement is accomplished.

Biomechanical data are often collected or represented in human-contrived mathematically or clinically relevant frames of reference. These frames of reference often obfuscate the researcher's vision of outcomes as the system under consideration does not necessarily behave in a manner where variabilities or differences in outcome data are expressed according to these reference frames (Daffertshofer et al., 2004). Differences in hammering movement are likely small making detection difficult if the movement is improperly represented. Under Principal Component Analysis (PCA), data sets which are of high dimension when expressed in human derived reference

Corresponding Author: Langenderfer Joseph E, School of Engineering and Technology, Central Michigan University, Mt. Pleasant, Michigan 48859, USA, Tel: 19897741911 
frames are reduced to the essential dimensions in which variance occurs. Higher variance explained with fewer components represents less complex movement when compared to smaller variance explained by a greater number of principal components (Federolf et al., 2013a, b). The number of one dimensional principal components for representing the variance represents a dimension describing the organization of the joint space degrees of freedom (Morrison and Newell, 2015) and classifies the movement in a manner beyond amplitude or time dependent measures by determining the number of independent, active dynamical degrees of freedom due to mechanical interactions within multi-joint kinematic chains and the action of inter-joint reflexes (Haken, 1996).

In order to better understand effects of hammer inertial properties, the purpose of this study was to use a PCA approach to calculate upper-extremity movement variance. The goal was to characterize the complexity of movement while subjects performed hammering with hammers commonly used in manual labor. In keeping with ideas from earlier studies, the hypothesis was that distinct differences in the hammering movement as indicated by the number of PCs required to explain movement variance would be found when subjects swung hammers with different properties.

\section{MATERIALS AND METHODS}

Subjects: 19 male right-hand dominant subjects (mean age: 24, range 22-29, height: mean $1.81 \mathrm{~m}$, SD $0.04 \mathrm{~m}$, mass: mean $80.7 \mathrm{~kg}$, SD $12.1 \mathrm{~kg}$ ) without history of upper-extremity orthopaedic or neuro-musculoskeletal injuries or surgeries and who had hammered nails on at least ten prior occasions were recruited from a university setting. All subjects provided informed written consent to participate in the study as required for approval from the institutional review board.

Experimental procedure: After subjects were familiarized with the nature of the experiment, they were allowed to practice swinging each hammer until comfortable. Then subjects swung four hammers (Fig. 1 a) while in a kneeling posture in order to drive nails according to an earlier described protocol (Balendra and Langenderfer, 2017). Three trials were performed with each hammer and hammer order was randomized across subjects. Nails were driven with commercially manufactured hammers commonly used in manual labor tasks with a standardized procedure where subjects swung the hammer one time at each nail while the nail was held in a jig. Hammer properties were measured prior to subject experimentation (Fig. 1b and
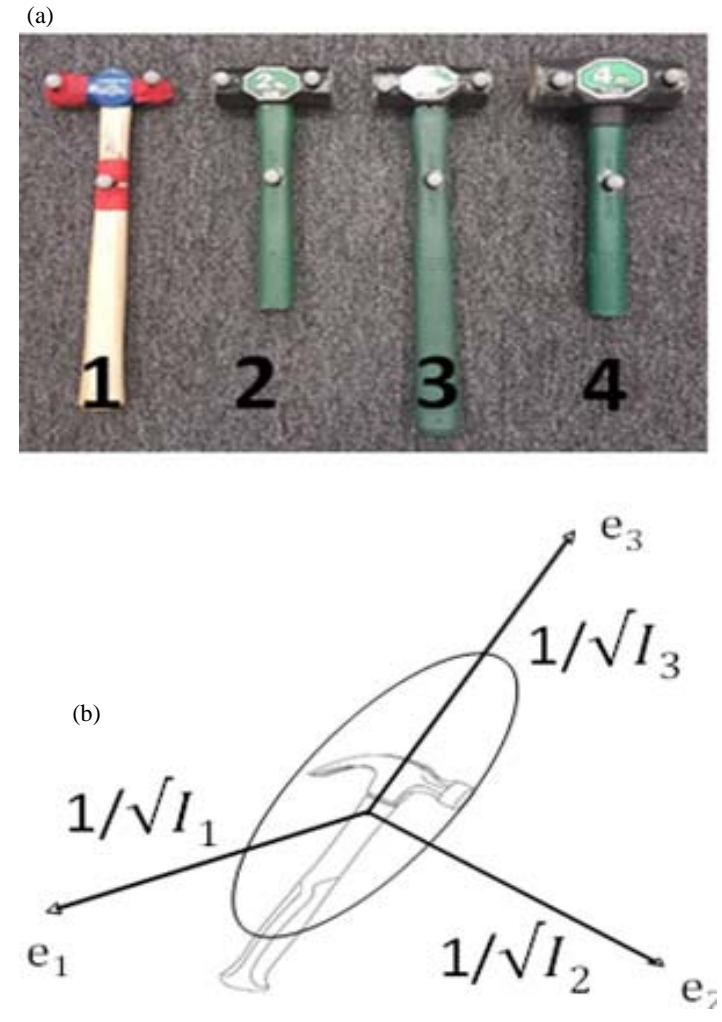

Fig. 1: (a) Hammers investigated in this study included common ball peen hammer $(1 \mathrm{lb})$ and $2 \mathrm{lb}$ (with 8 and 13 inch handles, respectively) and $4 \mathrm{lb}$ sledge hammers and (b) Inertia ellipsoid described by principal moments of inertia for hammer (shown in Table 1). Symmetry of inertia, S, calculated as: $\mathrm{S}=2 \mathrm{I}_{3} /\left(\mathrm{I}_{1}+\mathrm{I}_{2}\right)$ where $\mathrm{I}_{\mathrm{j}}$ are moments of inertia, $\mathrm{I}_{1}$ $>\mathrm{I}_{2}>\mathrm{I}_{3}$

Table 1). Transverse (swing weight) and lateral (spin weight) mass moment of inertia were calculated by treating the hammer as a simple pendulum and the polar (twist weight) mass moment of inertia was calculated using calibrated wire of known stiffness to suspend the hammer as a bifilar pendulum (Spurr et al., 2014). The principal moments of inertia $\mathrm{I}_{\mathrm{j}}$ were used to calculate hammer symmetry of inertia, $\mathrm{S}=2 \mathrm{I}_{3} /\left(\mathrm{I}_{1}+\mathrm{I}_{2}\right)$ where $\mathrm{I}_{1}>\mathrm{I}_{2}>\mathrm{I}_{3}$ (Carello and Wagman, 2009).

Passive retro-reflective markers were attached to thirteen landmarks of the upper-extremity and thorax and three points on the hammer (Fig. 1 and 2). The three-dimensional marker motion was recorded from hammer cocking, through swinging to nail strike with a optoelectronic motion capture system (Vicon, 12 camera T160, $100 \mathrm{~Hz}$ ). Data were post-processed to ensure correct marker identification and fill gaps for any occluded markers. 
Table 1: Hammer inertial properties

\begin{tabular}{lllll}
\hline Variables & 1 lb hammer & $2 \mathrm{lb}$ hammer & 2 lb hammer & $4 \mathrm{lb}$ hammer \\
\hline Mass [g] & 650 & 1100 & 1216 & 2090 \\
Swingweight $\left({\left.\mathrm{kg}-\mathrm{cm}^{2}\right)}^{\text {Spinweight }\left(\mathrm{kg}^{2} \mathrm{~cm}^{2}\right)}\right.$ & 58 & 53 & 124 & 80 \\
Twistweight $\left(\mathrm{kg}^{2}\right)$ & 55 & 46 & 117 & 57 \\
Volume $\left(\mathrm{kg}-\mathrm{cm}^{2}\right)^{-3 / 2}$ & 4 & 8 & 10 & 26 \\
Symmetry & $3.66 \times 10^{-2}$ & $3.05 \times 10^{-2}$ & $1.12 \times 10^{-2}$ & $1.11 \times 10^{-2}$ \\
\hline
\end{tabular}

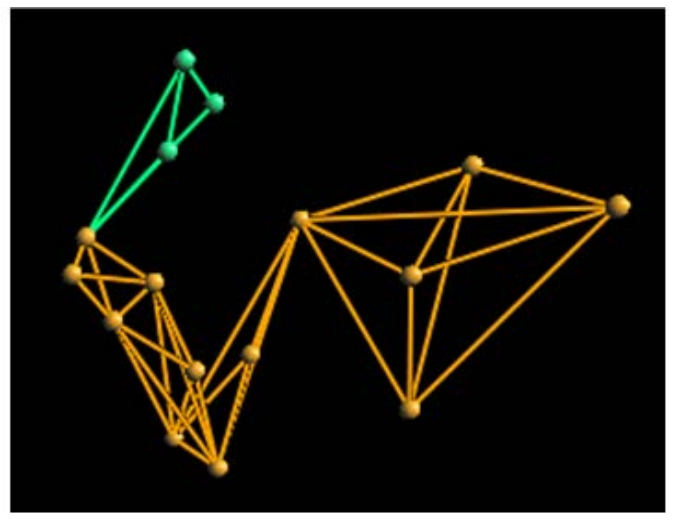

Fig. 2 : Movement of 13 markers attached to thorax and upper-extremity and 3 markers attached to hammers was recorded with Vicon as subjects swung hammers to strike nails. Markers on thorax and upper-extremity (orange) were spinous process of seventh cervical vertebra, xiphoid process, proximal clavicle at sterno-clavicular joint, bilateral acromion processes, bicep, medial and lateral humeral epicondyles, right forearm, radial and ulnar styloid processes, metacarpal phalangeal joints of middle and small fingers. Hammer markers (green) were attached to handle and hammer head

Data analysis: Each of the 3 trials from each subject for each hammer were inspected and linear length normalized (0-100\% of cycle) (Helwig et al., 2011) from initiation of movement defining start of the hammer cocking to nail strike. The linear length normalization ensured each trial possessed the same number of data points (i.e., 101) which is a requirement when assembling the matrix for PCA. For each trial, 48 spatial coordinates (3 dimensions of 16 markers on subject and hammer) defined a position vector $\mathrm{p}\left(\mathrm{t}_{\mathrm{i}}\right)$ at $1 \%$ time increments over the duration of the hammering movement. Marker data were then submitted to PCA (MATLAB R2016) using a procedure described previously (Federolf et al., 2013a, b). Like the earlier study, this study employed a normalization procedure in three steps. The purpose of the normalization was to minimize inter-subject differences in anthropometry and associated subject position while retaining hammering motion variability. The three steps were: the calculation of a mean position $\mathrm{p}_{\text {mean }}$ for each trial which was subtracted from that trial's marker data, the calculation of a vector norm $\mathrm{d}\left(\mathrm{t}_{\mathrm{i}}\right)$ of the mean centered marker data and lastly the mean centered marker data was divided by the mean vector norm $d_{\text {mean }}$ to complete the process:

$$
\mathrm{P}_{\text {norm }}\left(\mathrm{t}_{\mathrm{i}}\right)=\left(\mathrm{p}\left(\mathrm{t}_{\mathrm{i}}\right)-\mathrm{p}_{\text {mean }}\right) / \mathrm{d}_{\text {mean }}
$$

The normalized position vectors $p_{\text {norm }}\left(t_{i}\right)$ for all subjects were then concatenated for PCA resulting in a 5757 by 48 dimension matrix ( 19 subjects x 3 trials $x 101$ linear length normalized time points by 16 markers $\mathrm{x} 3$ cartesian coordinates) describing the hammering movement.

Quantification of movement variability: As is well described, the PCA performed on the covariance matrix of position vector data yielded eigenvectors describing orthogonal Principal Components $\left(\mathrm{PC}_{\mathrm{j}}\right.$ ) and Eigen Values $\left(\mathrm{EV}_{\mathrm{j}}\right)$ (Deluzio et al., 1997, Federolf et al., 2013a, b). By rank ordering the eigenvalues according to the amount of variance explained (i.e., normalizing each eigenvalue by the sum of all eigenvalues), the contribution of each principal component in explaining variance in the original marker data can be calculated. Principal movements $\mathbf{P M}_{\mathrm{j}}$ can be reconstructed in the original Cartesian space which are the normalized position vectors $\mathrm{p}_{\text {norm }}\left(\mathrm{t}_{\mathrm{i}}\right)$ projected onto the principal components $\mathrm{P}_{\mathrm{cj}}$ after rescinding the normalization procedure:

$$
\mathrm{PM}_{\mathrm{j}}=\mathrm{p}_{\text {mean }}+\mathrm{a}_{\mathrm{j}} \mathrm{d}_{\text {mean }} \mathrm{P}_{\text {norm }}\left(\mathrm{t}_{\mathrm{i}}\right) \mathrm{PC}_{\mathrm{j}}
$$

To interpret the principal movements and allow for a qualitative and visual understanding of how each contributes to the hammering movement, these principal movements can be animated or plotted (Fig. 3). As the contribution of individual PCs to explaining variance can generally be small, especially for higher ordered components that explain less variance, the amplification factor $\mathrm{a}_{\mathrm{j}}$ allows for a visual assessment of the principal movement. In an analogous manner as eigenvalues explain variance of each principal component across all trials submitted to PCA, similar measures of variance explained by each PC were calculated within each trial. The variance $\mathrm{s}_{\mathrm{j}}{ }^{2}$ of the normalized position vectors projected on to the PCs determines the contribution of 
J. Eng. Applied Sci., 15 (6): 1515-1521, 2020

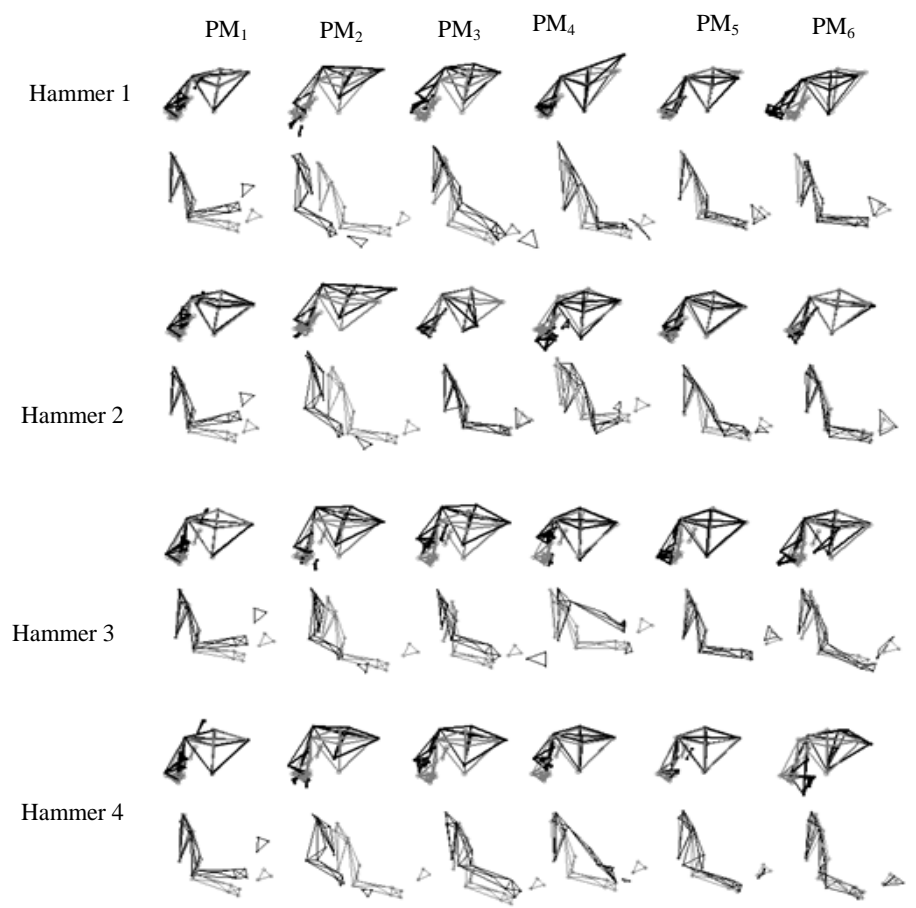

Fig. 3: Visualization of the first 6 principal movements $\mathrm{PM}_{\mathrm{j}}$. Top rows are frontal view and bottom rows are sagittal view for each of 4 hammers, respectively. Gray lines and markers represent the mean and black lines and markers represent the largest deviation (i.e., largest RMS difference during the hammering movement) in the direction of principal component $\mathrm{PC}_{\mathrm{j}}$. Deviations were amplified for effective visualization of subtle movements: $\mathrm{a}_{1}=1$ for $\mathrm{PM}_{1}, \mathrm{a}_{2}=5$ for $\mathrm{PM}_{2}, \mathrm{a}_{3}=10$ for $\mathrm{PM}_{3}, \mathrm{a}_{4}=\mathrm{a}_{5}=20$ for $\mathrm{PM}_{4}$, and $\mathrm{PM}_{5}$, and $\mathrm{a}_{6}=30$ for $\mathrm{PM}_{6}$

each PC to within trial variance (Federolf et al., 2013a ,b). Similar to the manner in which eigenvalues are normalized in order to determine their relative contribution to explaining variance, the $s_{j}^{2}$ were normalized by dividing by the sum of all $\mathrm{s}_{\mathrm{j}}^{2}$ for a subject. From these normalized variances, the cumulative normalized variance was calculated to represent the percentage of variance explained by a certain number of PCs for a trial. The cumulative normalized variance can be interpreted as a measure of movement complexity. That is if a greater number of principal components are required to explain the movement at a certain variance level, then the movement is more complex (Federolf et al., 2013a, b). At each Principal Movement $\mathrm{PM}_{\mathrm{i}}$, the cumulative normalized variance between hammers was assessed with ANOVA and pairwise differences determined with Tukey's Honest Significant Difference test.

Sensitivity analysis: To assess how sensitive results are to individual subjects, a leave-one-out cross validation was conducted consecutively with data from all subjects. PC vectors were calculated when the data of each subject was left out from the PCA. A dot product was calculated between each of the leave-one-out PC vectors and the all-subject PC vector. When each subject was left out the percent variance explained was re-calculated by projecting each subject's normalized position vectors onto the leave-one-out PC vectors. Differences in percent variance explained were calculated between the all-subject results and the leave-one-out results to obtain a measure of the average difference in percent variance explained for each of the first $10 \mathrm{PM}_{\mathrm{j}}$ when a subject was left-out.

\section{RESULTS AND DISCUSSION}

Contributions of the first six PM to the overall hammering movement can be observed (Fig. 3). For all hammers, the most dominant movement, $\mathrm{PM}_{1}$ was elbow flexion-extension. For hammer 1, $\mathrm{PM}_{1}$ explained $77.4 \%$ of movement variance whereas $\mathrm{PM}_{1}$ explained $76.2 \%$, 78.5 and $76.0 \%$ of variance for hammers 2,3 and 4 , respectively. For all $\mathrm{PM}_{\mathrm{i}}$ the percentages of explained variance of the hammering movement are depicted in Fig. 4 and 5. For all hammers, $\mathrm{PM}_{2}$ was movement of the thorax in both a translation and rotation along and about, respectively an anterior-posterior axis. For hammers 1,3 and $4, \mathrm{PM}_{3}$ was a rotation of the involved shoulder in relation to the non-involved shoulder but for hammer 2 , 

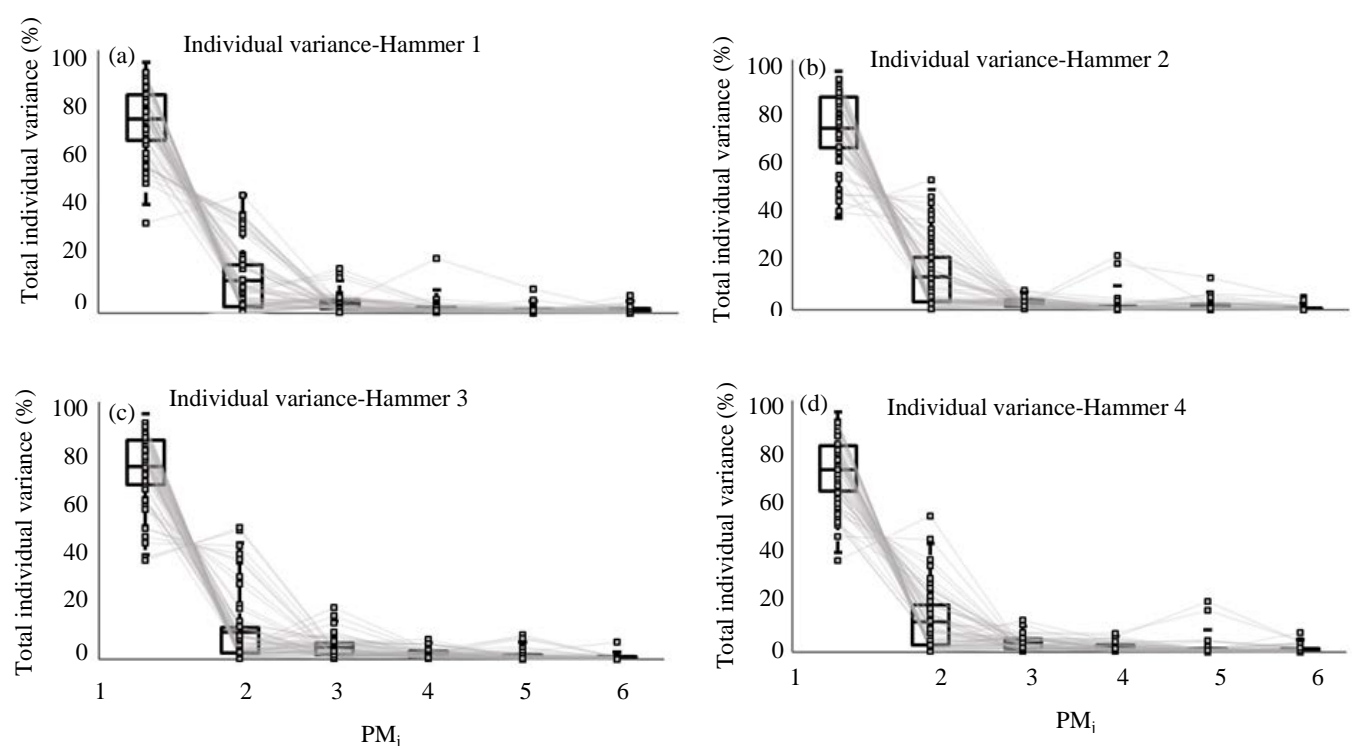

Fig. 4(a-d): Normalized variance $\mathrm{s}^{2}$ for the first six principal movements of each subject for the four hammers represented as box plot of mean and interquartile range, whiskers are $95 \%$ confidence intervals. Individual subject results for each trial are the gray points connected with gray lines

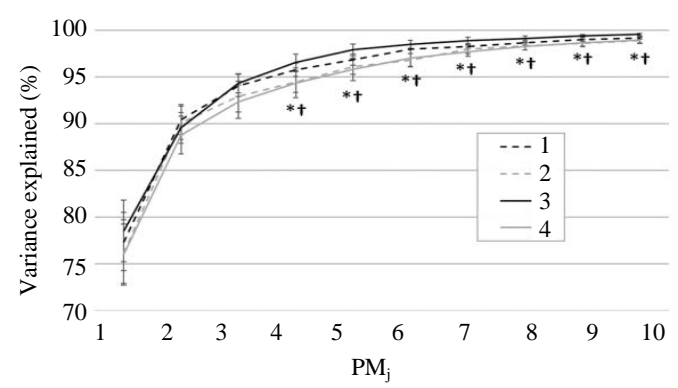

Fig. 5: Cumulative normalized variance explained by the first ten principal movements $\mathrm{PM}_{\mathrm{j}}$ for the four hammers. Error bars are standard error. For a given variance level $>90 \%$ hammers with long handle required fewer principal movements to explain the overall hammering movement. Significant differences comparing hammers are represented as $2-3 *$, 3-4 $†$

$\mathrm{PM}_{3}$ was a superior-inferior movement of the non-involved shoulder. $\mathrm{PM}_{4}$ can be interpreted as an anterior-posterior movement of the arm and hammer with associated shoulder movement that differed between hammers. For hammers 1 and 2, the associated $\mathrm{PM}_{4}$ shoulder movement was translation of the non-involved shoulder in the superior-inferior direction and a rotation of the involved shoulder in relation to the non-involved shoulder, respectively. For hammers 3 and 4, there were no $\mathrm{PM}_{4}$ associated movements of the shoulders. $\mathrm{PM}_{5}$ was shoulder internal-external rotation associated with positioning the hand and hammer to accurately strike the nail. For hammer $1, \mathrm{PM}_{6}$ can be interpreted as shoulder abduction-adduction but for hammer $2 \mathrm{PM}_{6}$ was a positioning of the elbow. For hammers 3 and $4, \mathrm{PM}_{6}$ was shoulder abduction-adduction with thorax leaning in same direction as abduction-adduction movement. Higher order principal movements contribute much smaller amounts to total movement variance and thus are difficult to interpret.

The normalized variance $s^{2}$ for the four hammers (Fig. 4) represents the distribution of strategies selected for performing the hammering movement and allows for understanding of the structure of movement variance. Individual trial results are informative for understanding how subjects swung the hammers according to the principal movements described, here and show considerable inter-subject differences. For hammer 2, $\mathrm{PM}_{3}$ was only selected as a movement strategy by a few subjects. In general from the structure of the movement variance it can be observed that most subjects swung the hammers in a way that followed the expected movement strategy as indicated by trials where lower $\mathrm{PM}_{\mathrm{j}}$ explained more variance in movement (i.e., negative slope for the lines connecting $\mathrm{PM}_{\mathrm{i}}$ ). For trials that followed the mean expected pattern of negative slopes, there are distinct differences in the slopes of the lines connecting $\mathrm{PM}_{\mathrm{i}}$ indicating a considerable distribution of movement strategies selected by subjects for these trials. Furthermore, some subjects selected alternate strategies that differed considerably from the generally expected strategy as shown by trials with lines of positive slope indicating a lower $\mathrm{PM}_{\mathrm{j}}$ explained less variance than a higher $\mathrm{PM}_{\mathrm{j}}$. 
Analysis of the cumulative normalized variance reveals that, on average, across all subjects, three $\mathrm{PM}_{\mathrm{i}}$ explained at least $90 \%$ of the movement variance for all hammers (Fig. 5). For the first three principal movements: $\mathrm{PM}_{1}, \mathrm{PM}_{2}$ and $\mathrm{PM}_{3}$, no differences in mean cumulative variance were detected between hammers. In order to explain 95\% variance, four $\mathrm{PM}_{\mathrm{j}}$ were required to explain movement of the long handled hammer hammers, while for the short handle hammers a fifth $\mathrm{PM}_{\mathrm{j}}$ was required. The result that hammers with long handles required fewer $\mathrm{PM}_{\mathrm{j}}$ to explain the movement variance continued at higher cumulative variance levels indicating a smaller movement dimension for long handle hammers. The variance explained by $\mathrm{PM}_{4}(\mathrm{p}=0.02)$ and $\mathrm{PM}_{5}$ through $\mathrm{PM}_{10}$ (all $\mathrm{p}<0.01$ ) were less for hammer 2 (short handle) compared to hammer 3 (long handle). When comparing hammers 3 and 4 , the variance explained by $\mathrm{PM}_{4}$ was less for the short handle hammer (hammer 4) ( $\mathrm{p}=0.02)$. Likewise, $\mathrm{PM}_{5}$ through $\mathrm{PM}_{10}$ explained less variance for the short handle hammer (hammer 4) compared to the long handle hammer 3 (all $\mathrm{p}=0.01$, or smaller).

The leave-one-out cross validation sensitivity analysis revealed that removing one subject from the PCA calculation yielded similar PC vectors as when data from all subjects were analyzed. The dot product results ranged from $0.9998 \pm 0.0004$ for $\mathrm{PC}_{1}$, to $0.9140 \pm 0.2137$ for $\mathrm{PC}_{10}$ (mean $\pm \mathrm{SD}$ ). The average absolute difference in percent variance explained between the all-subject results and the leave one out results for each of the first 10 PM's was $0.18 \%$ with a range from $0.57 \%$ for $\mathrm{PM}_{1}$ to $0.04 \%$ for $\mathrm{PM}_{10}$, indicating results are robust to removal of individual subjects.

This study was designed to quantify differences in hammering movements while describing the role of hammer properties. The hypothesis that movement variance is affected by hammer properties was supported as longer handle hammers required fewer principal components to explain variance. Differences in movement variance across hammers were quite small indicating that subjects generally swung the hammers in a similar manner as evidenced by the lack of differences at lower-ordered principal movements explaining <95\% variance. At levels beyond 95\% variance the short handle hammers (hammers 2 and 4) required orthogonal movements which were not present to the same extent for long handle hammer (hammer 3). Subjects selected differing movement strategies consisting primarily of elbow flexion but also thorax movements to accomplish the hammering task and the strategies were different between hammers. A greater number of $\mathrm{PM}_{\mathrm{j}}$ required for explaining variance and thus greater dimension for the shorter handle hammers can be interpreted as reflecting a strategy for coordinated recruitment of mechanical degrees of freedom resulting in more complex movement (Federolf et al., 2013a, b). The more complex movement for shorter handle hammers which are generally not as commonly utilized, may have been related to reduced subject familiarity from lack of experience associated with performance of a not as well known task and consequently greater freezing of degrees of freedom resulting in a less coordinated movement (Bernstein, 1967). However, a different explanation is possible as some subjects adopted a movement pattern involving the thorax extension but also lateral bending $\left(\mathrm{PM}_{2}\right)$ as alternative to upper-extremity movement as the shorter handles required reaching towards the nail to achieve the hammering task. Movement dimension and associated variability is representative of compensatory mechanisms which allow for performance but have been shown to indicate decreased stability of movement (Dona et al., 2009). As such, a smaller movement dimension found for long handle hammers represents a more efficient movement from greater freeing and coordination of mechanical degrees of freedom with less complex movement, and reduced risk of injury to musculoskeletal tissues (Mitra et al., 1998).

The dimension and associated complexity of movement may be useful in understanding differences in motor control and loads on musculoskeletal tissues. For example, the inclusion of the $\mathrm{PM}_{5}$ to achieve the $95 \%$ variance level for short handle hammers indicates shoulder internal-external rotation which is not present to the same extent as for long handle hammers. $\mathrm{PM}_{6}$ which involved an abduction-adduction movement for thorax and entire upper-extremity for hammers 3 and 4 but not other hammers can be interpreted as a more complex movement for the thorax which may result in lumbar loading from abduction-adduction movement of thorax. Even though there were no significant differences across hammers for the first three $\mathrm{PM}_{\mathrm{j}}$, the movement strategies selected were subject-specific and hammer specific as indicated by distributions of inter-trial variance explained by the $\mathrm{PM}_{\mathrm{j}}$. Across subjects, the lighter, long handle hammer (1) resulted in all but one subject swinging the hammer where $\mathrm{PM}_{1}$ (elbow flexion) was the dominant movement. For the other hammers, subjects demonstrated greater differences in the relative importance of $\mathrm{PM}_{1}$ and $\mathrm{PM}_{2}$ in contributing to the overall movement, indicating less consistency in strategies. The lack of consistency may be related to decreased familiarity or ease of use of these hammers which are less commonly implemented for driving nails. The differences in variance explained by $\mathrm{PM}_{1}$ and $\mathrm{PM}_{2}$ do not occur in isolation. When $\mathrm{PM}_{1}$ contributed less to variance, $\mathrm{PM}_{2}$ tended to contribute more to variance. For most subjects, elbow flexion was the dominant movement but for a few subjects the thorax was nearly as dominant and in a few trials, more dominant. This result highlights that hammers are swung with considerable differences in contributions of elbow flexion and thorax movement in accomplishing the task. 
Previously, hammers with greater symmetry of inertia have been found to be less effective (Fitzpatrick, et al., 2012). Although, the peg pounding hammering task in this earlier work was similar, it was not as functional as the nail driving tested, here and employed toy hammers, i.e., not hammers common for manual labor. Therefore, subjects were not challenged to the same extent as the current study. As a practical matter for commonly used hammers, because the handle length is the primary means by which the symmetry of inertia is affected, the use of these hammers meant that symmetry was not controlled independent of handle length. Such methodological difference may have led to the lack of finding any affect of hammer symmetry of inertia on movement variance. In terms of kinetics, longer handle length could be expected to influence the interaction force and torque between handle and hand and likewise at upper-extremity joints in a manner similar to how hammer mass has been shown to increase joint moments (Balendra and Langenderfer, 2017). However, the effect of handle length on joint moments has not been specifically investigated.

\section{CONCLUSION}

Principal movements were different between hammers and subjects used different movement strategies as indicated by a different structure of the movement variance between hammers. Differences in the explained variance of hammering movements demonstrate a more complex movement for short handle hammers. This research provides documentation of techniques for investigating the potential effects of object properties on movement and potentially other biomechanical factors including joint moments and forces. Such considerations are important, not just for hammers but other manipulated or swung objects including tools, prosthetic limbs and implements utilized in sport.

\section{ACKNOWLEDGEMENTS}

This study was made possible and supported by equipment purchased under NSF-CBE-MRI 1337511. Sponsors had no role in: study design, data collection or analysis, interpretation of data in manuscript writing or decision to submit the manuscript for publication.

\section{REFERENCES}

Balendra, N. and J.E. Langenderfer, 2017. Effect of hammer mass on upper extremity joint moments. Applied Ergon., 60: 231-239.

Bernstein, N., 1967. The Co-Ordination and Regulation of Movements. Pergamon Press, Oxford, UK., ISBN: 9780080119403, Pages: 196.
Carello, C. and J.B. Wagman, 2009. Mutuality in the Perception of Affordances and the Control of Movement. In: Progress in Motor Control: A Multidisciplinary Perspective, Sternad, D. (Ed.). Springer, Boston, Massachusetts, ISBN: 978-0-387-77063-5, pp: 273-292.

Cote, J.N., D. Raymond, P.A. Mathieu, A.G. Feldman and M.F. Levin, 2005. Differences in multi-joint kinematic patterns of repetitive hammering in healthy, fatigued and shoulder-injured individuals. Clin. Biomech., 20: 581-590.

Daffertshofer, A., C.J.C. Lamoth, O.G. Meijer and P.J. Beek, 2004. PCA in studying coordination and variability: A tutorial. Clin. Biomech., 19: 415-428.

Deluzio, K.J., U.P. Wyss, B. Zee, P.A. Costigan and C. Serbie, 1997. Principal component models of knee kinematics and kinetics: Normal vs. pathological gait patterns. Hum. Mov. Sci., 16: 201-217.

Dona, G., E. Preatoni, C. Cobelli, R. Rodano and A.J. Harrison, 2009. Application of functional principal component analysis in race walking: An emerging methodology. Sports Biomech., 8: 284-301.

Federolf, P., L. Roos and B.M. Nigg, 2013b. Analysis of the multi-segmental postural movement strategies utilized in bipedal, tandem and one-leg stance as quantified by a principal component decomposition of marker coordinates. J. Biomech., 46: 2626-2633.

Federolf, P.A., K.A. Boyer and T.P. Andriacchi, 2013a. Application of principal component analysis in clinical gait research: Identification of systematic differences between healthy and medial knee-osteoarthritic gait. J. Biomech., 46: 2173-2178.

Fitzpatrick, P., J.B. Wagman and R.C. Schmidt, 2015. Alterations in movement dynamics in a tool-use task the role of action-relevant inertial tool properties. $\mathrm{Z}$. Psychologie, 220: 23-28.

Haken, H., 1996. Principles of Brain Functioning: A Synergetic Approach to Brain Activity, Behavior and Cognition. Springer, New York, USA., ISBN: 9783540589679, Pages: 347.

Helwig, N.E., S. Hong, E.T. Hsiao-Wecksler and J.D. Polk, 2011. Methods to temporally align gait cycle data. J. Biomech., 44: 561-566.

Mitra, S., P.G. Amazeen and M.T. Turvey, 1998. Intermediate motor learning as decreasing active (dynamical) degrees of freedom. Hum. Mov. Sci., 17: 17-65.

Morrison, S. and K.M. Newell, 2015. Dimension and complexity in human movement and posture. Nonlinear Dyn. Psychol. Life Sci., 19: 395-418.

Petric, T., C.S. Simpson, A. Ude and A.J. Ijspeert, 2017. Hammering does not fit fitts' law. Front. Comput. Neurosci., Vol. 11, 10.3389/fncom.2017.00045

Spurr, J., S. Goodwill, J. Kelley and S. Haake, 2014. Measuring the inertial properties of a tennis racket. Procedia Eng., 72: 569-574. 